Results CHD risks were estimated in 1920 (FRS 1998) and 2046 (FRS 2002) of HIV subjects while CVDs risk were evaluated in 1927 subjects. Median ages and percentage of male were 44 years and 81.4\% (FRS 1998, 2002 and FRS 2008). Approximatly 90\% were receiving NNRTI+ NRTI and just 13\% and $10 \%$ were taking tenofovir and a protease inhibitor. Beside $11 \%$ were smokers while $14 \%$ as diabetic. Dyslipidemia was seen at least in $45 \%$ of population. Equally $8 \%$ had CD4 count $<200$ cells $/ \mathrm{mm}^{3}$ and 9\% hepatitis C. Importantly $10 \%$ and $4 \%$ had intermediate and high CHD risks (FRS 1998) and 6.6\% and $3.3 \%$ with intermediate and high risks for CHD based on FRS (2002) intermediate and high risk of CVDs was prevalent in while $39 \%$ and $8 \%$ of HIV subjects. Among all studied variables, higher total cholesterol levels and older age were the strong risk predictors for CHD and CVDs $(\mathrm{p}<0.05)$.

Conclusions We found a high prevalence of dyslipidemia while the CHD risks measured by the Framingham scales 1998 and 2002 were low. Notably CVDs risk was high thus further investigations as well preventive management should be prioritised in this population.

\section{P16.29 PREVALENCE OF METABOLIC RISK FACTORS IN HIV- INFECTED POPULATION UNDER ANTIRETROVIRAL THERAPY IN NORTHERN MALAWI}

${ }^{1} \mathrm{CC}$ Chung ${ }^{*},{ }^{1} \mathrm{HH}$ Lin, ${ }^{2} \mathrm{CC}$ Mtika, ${ }^{3} \mathrm{~J}$ Wu. ${ }^{1}$ National Taiwan University, Institute of Epidemiology and Preventive Medicine; ${ }^{2}$ Mzuzu Central Hospital (Malawi); ${ }^{3}$ Luke International in Malawi

\subsection{6/sextrans-2015-052270.576}

Introduction With increased availability of antiretroviral therapy (ART), the life expectancy of people with human immunodeficiency virus (HIV) infection is expected to improve substantially. On the other hand, life-long ART may be associated with increased obesity, diabetes, and hypertension. The situation of obesity, diabetes, and hypertension in HIV-infected population in Malawi is unclear.

Methods We conducted a cross-sectional study including adult HIV-infected patients under ART treatment in a teaching hospital from northern Malawi. Trained field workers performed anthropometric measurements, blood pressure exam, and finger prick test for blood glucose. We followed the WHO and International Diabetes Federation (IDF) criteria to define obesity, hypertension, and diabetes. We compared the prevalence of metabolic risk factors in our study population to that in the 2009 STEPwise approach to Surveillance (STEPS) survey in Malawi.

Results The study included 410 participants (20.97\% males). The prevalence of overweight $(25 \leq \mathrm{BMI}<30)$ and obesity (BMI $\geq 30$ ) was $22.9 \%$ (95\% CI: $18.9-26.9 \%$ ) and $10.0 \%$ (95\% CI: 7.1-12.9\%), respectively. The prevalence was higher than that in the STEPS survey (21.9\% for overweight and $4.6 \%$ for obesity). Notably the situation of overweight and obesity was much worse in females $(26.8 \%$ and $12.3 \%)$ than in males $(8.2 \%$ and $1.2 \%)$. Old age (OR: 1.064 per year, $\mathrm{p}=0.0016)$ and alcohol drinking (OR: 7.309, $\mathrm{p}=0.0005$ ) were significant associated with obesity. The prevalence of diabetes or impaired fasting glucose (20.2\%, 95\% CI: 16.3-24.1\%) was higher than that in STEPS survey $(9.8 \%)$. The overall prevalence of hypertension was $18.1 \%$ (95\% CI: $14.3-21.8 \%$ ), lower than that in the STEPS survey (32.9\%).

Conclusion Our study provides an initial assessment of the burden of metabolic risk factors among HIV-infected population under long-term ART treatment in Malawi. The high prevalence of metabolic risk factors in this population is concerning. Longterm impact on the burden of noncommunicable diseases warrants further investigation.

Disclosure of interest statement This study was funded by Luke international belongs Pingtung Christian hospital. And that was leaded by National Taiwan University for academic research.

\section{P16.30 EPIDEMIOLOGY OF ANAL INFECTION IN HIV INFECTED PATIENTS ATTENDING A SEXUALLY TRANSMITTED INFECTION CLINIC IN BRAZIL}

Boldrini Nat*, LP Bondi, LC Spano, LB Freitas, AE Miranda. Post-Graduation Program in Infectious Diseases, Universidade Federal Do Espirito Santo

\subsection{6/sextrans-2015-052270.577}

Introduction Anal squamous cell carcinoma is rare in the general population but certain populations, such as persons with HIV, are at increased risk. High-risk populations can be screened for anal cancer using strategies similar to those used for cervical cancer. The objective of this study was determinating the prevalence, genotype distribution and risk factors associated with anal HPV infection among persons of both sexes with HIV attending a STI clinic in Espirito Santo, Brazil.

Methods Cross sectional study assessing HIV-infected persons. A comprehensive survey was administered that included a demographic and behavioural assessment. Anal specimens were collected for cytology and HPV screening using Polymerase Chain Reaction.

Results A total of 169 patients completed the study, 122 female and 47 male, mean age was 40.3 years, $81.4 \%$ lived in Vitoria, the average education was 8.6 years, $48.8 \%$ were married, $42.1 \%$ had between 5 and 20 sexual partners, $25.4 \%$ initiated sexual activity before age $15.72 \%$ reported anal sexual activity, $58.3 \%$ had a history of STI, the most frequent being condyloma acuminata (25.4\%) followed by syphilis (9\%). 31.6\% knew to be HIV positive for more than 10 years, $65.1 \%$ had an undetectable viral load, only $3.5 \%$ had CD4 below 200 cells and $82.9 \%$ were taking HAART. Anal cytology in both sexes had a $13.2 \%$ prevalence changes. The prevalence of HPV infection of any type was $71 \%$, and high-risk HPV types were $52.4 \%$. The HPV types most frequent high-risk types were: 16, 51 and 53. 37.3\% had multiple HPV types.

Conclusions Anal HPV is common among HIV-infected persons attending this STI clinic, repeated annual cytology screening for HIV-infected, particularly for those with increased immunosuppression, anal HPV, history of the other STIs, or abnormal cervical cytology will increase the likelihood of detecting AIN 2-3.

Disclosure of interest statement There is no conflict of interest.

\section{P16.31 IMPROVING TIMELY HIV RESULTS IN KEY POPULATIONS: RAPID TEST ANTIBODY EVALUATION FOR HIV DIAGNOSIS IN GUATEMALA, 2012-2013}

${ }^{1}$ E Arana Flora, ${ }^{2} \mathrm{~L}$ Castillo-Signor, ${ }^{2} \mathrm{P}$ Marchorro, ${ }^{3} \mathrm{~V}$ Girón, ${ }^{2} \mathrm{~A}$ Lopez, ${ }^{2} \mathrm{C}$ Escobar, ${ }^{1} \mathrm{C}$ Vargas, ${ }^{1} \mathrm{R}$ Mendizabal-Burastero, ${ }^{1} \mathrm{~S}$ Morales-Miranda*. ${ }^{1} \mathrm{HIV}$ Unit, Centers for Health Studies, Del Valle University; ${ }^{2}$ National Health Laboratory, Ministry of Health, Guatemala; ${ }^{3}$ HIV National Program, Ministry of Health, Guatemala

\subsection{6/sextrans-2015-052270.578}

Introduction Guatemala has a concentrated HIV epidemic among key populations; in 2003, the first rapid test validation in Central America was performed. A national HIV testing algorithm 
with a rapid test and a second test using an enzyme immunoassay (EIA) was developed (average turnaround time of 2 weeks). Given the changes in the market since 2003, we present results of a second rapid test validation to update the Guatemalan algorithm.

Methods Validation was performed in 2012-2013, evaluating a total of 10 rapid tests in two phases: 1) with serum, HIV-negative samples were obtained from blood banks, and positive samples from HIV care units; 2) with whole blood, negative samples were obtained from antenatal care services and positive samples from HIV care units. We tested 452 serum samples [244 HIV+, 208 HIV-] and 432 whole blood samples (10 HIV positive, 422 HIV negative) using two EIA (Roche ELECSYS HIV Combi and Abbott AXSYM HIV Ag/Ab Combo). Discordant results were evaluated with HIV-1 Western Blot. For fourth generation rapid tests, only antibody was evaluated. Data was analysed using OpenEpi.

Results Six HIV rapid tests were included for both phases. All tests (except Double Check Gold) reported sensitivity higher than $99 \%$ and specificity higher than 95\%. Determine HIV 1/2 and $\mathrm{HIV} \mathrm{Ag} / \mathrm{Ab}$ reported the highest results for sensitivity (100\%). Hexagon HIV (100\%) and Accu-Tell (99.5\%) reported the highest results for specificity. All rapid tests that also included HIV-2 detection, showed cross-reactivity, ranging from $37 \%$ of HIV cases for Anarapid HIV 1/2/O Tri-line to 15\% for Rapid HIV 1/2/O Tri-Line.

Conclusion Our results show that Determine HIV $1 / 2$ and HIV $\mathrm{Ag} / \mathrm{Ab}$ should be recommended as a screening test while Hexagon HIV and Accu-Tell should be recommended as second test. Rapid test validation provides valuable information for on-site confirmation of reactive results improving diagnosis turnaround time.

Disclosure of interest statement We declare that we have no conflicts of interest.

\section{P17 - HIV testing, treatment and care}

\section{P17.01 PERFORMANCE EVALUATION OF THE GENEXPERT HIV-1 QUANT ASSAY FOR DETECTION OF HIV-1 IN PLASMA}

L McNally, A Carrera, J Sherring, PH Cunningham. NSW State Reference Laboratory for HIV, St Vincent's Hospital Sydney Limited, Kirby Institute UNSW

\subsection{6/sextrans-2015-052270.579}

Introduction The HIV-1 Quant Assay performed on the GeneXpert ${ }^{\circledR}$ Instrument Systems, is designed for the rapid quantitation of HIV-1 in human plasma with an analytical range of 40 to 10,000,000 copies/mL for HIV-1 Group M subtypes A, C, D, $\mathrm{AE}, \mathrm{F}, \mathrm{G}, \mathrm{H}, \mathrm{AB}, \mathrm{AG}, \mathrm{J}, \mathrm{K}$ and Groups $\mathrm{N}$ and $\mathrm{O}$. Testing is performed in a single-use disposable GeneXpert cartridges that hold the real-time reverse transcriptase polymerase chain reaction (RT-PCR) reagents and host the RT-PCR processes. This study assessed the performance of the system in routine plasma.

Methods To date, a total of 130 plasma samples have been tested over the analytical range and compared to a benchmark real time PCR system. Seventy four samples (56.9\%) were of a known subtype comprising of subtype B (37.6\%), AE (7.7\%), C (4.6\%) AG (1.5\%) and mixed (4.6\%). Additional samples consisting of an external quality control samples run over multiple days, and samples with HIV-1 RNA not detected or below the lower limit of were also tested to assess performance.
Results Overall the HIV-1 Quant Assay performed on the GeneXpert ${ }^{\circledR}$ Instrument Systems correlated with the routine analytical platform $(\mathrm{r} 2=0.9333)$. Samples ranged undetectable $(16$, $8.8 \%)$, below the benchmark test lower limit of detection $(<20$ $\mathrm{cpy} / \mathrm{ml})(10,7.9 \%)$, low range $(20-5,000)(43,33.1 \%)$, medium $(5,000-50,000)(24,18.5 \%)$ and high range $(>50,000 \mathrm{cpy} / \mathrm{ml})$ $(29,22.3 \%)$. Thirteen samples $(10 \%)$ were invalid as a result of insufficient sample. Samples in the lower analytical range $<1,000 \mathrm{cpy} / \mathrm{ml}$ showed little variance when compared with the Roche (CAP/CTM) assay using Bland-Altman correlation analysis. Reproducibility was assessed in the high, medium and low range within 1-2SD of mean. Sixteen replicates of a commercial external control showed very good reproducibility.

Conclusion The HIV-1 Quant Assay performed on the GeneXpert ${ }^{\circledR}$ Instrument Systems correlated with a commonly used HIV RNA test in plasma and offered significant workflow advantages. The system has a small footprint and requires no further consumables other that the single-use test cartridges. Further studies are planned to fully assess the assay performance.

Disclosure of interest statement No conflict of interest to declare.

\section{P17.02 ORAQUICK $®$ IN-HOME HIV TEST KIT IN PERU: AVAILABILITY AND ACCEPTABILITY AMONG MEN WHO HAVE SEX WITH MEN AND TRANSGENDER WOMEN}

${ }^{1}$ Maria Jose Bustamante*, ${ }^{1,2}$ Kelika A Konda, ${ }^{2}$ Segundo $\mathrm{R}$ León, ${ }^{3}$ Gino Calvo, ${ }^{4}$ Javier Salvatierra, ${ }^{5}$ Brandon Brown, ${ }^{2}$ Carlos F Caceres, ${ }^{2}$ Jeffrey D Klausner. ${ }^{1}$ Unit of Health, Sexuality and Human Development, and Laboratory of Sexual Health, Universidad Peruana Cayetano Heredia, Lima, Peru; '2Program in Global Health, Department of Medicine, University of California Los Angeles, Los Angeles CA, USA; ${ }^{3}$ Epicentro Salud, Lima, Peru; ${ }^{4}$ Barton Health Center, Health Directorate of Callao, Lima, Peru; ${ }^{5}$ Program in Public Health, University of California Irvine, Irvine CA, USA

\section{$10.1136 /$ sextrans-2015-052270.580}

Background In Peru an estimated 70\% of people who are HIV positive do not know their status. Knowing one's HIV status is critical in HIV prevention. Oraquick ${ }^{\circledR}$ in-home HIV test is the first rapid HIV self-test U. S FDA-approved for home use. We aimed to assess its availability and the willingness of men who have sex with men (MSM) and transgender women (TW) to use it in Peru.

Methods Four Pharmacy chains in Peru were surveyed to ascertain commercial availability of the Oraquick ${ }^{\circledR}$ in-home HIV test kits. High-risk MSM and TW who attended either of two STI clinics in Lima from June 2013 to May 2014 were surveyed. Data on demographics and willingness to use Oraquick ${ }^{\circledR}$ IN HOME HIV TEST kit were collected using an interviewer administered computer-based questionnaire.

Results The Oraquick ${ }^{\circledR}$ Rapid HIV-1/2 test kit was available for purchase for home use by $4(100 \%)$ pharmacy chains, 3 in Lima and one in northern Peru. The average test kit cost was 54 soles (18 USD); kits were available to clients 18 years or older for over-the-counter purchase. Of the 137 interviewed survey participants, $85 \%(\mathrm{n}=117)$ reported they would use a rapid home HIV self-test at least twice yearly. Respondents reported willingness to pay up to 21 soles (approximately 7 USD) for the test and in the event of positive results, to do the confirmatory blood test in a clinic. Also, $78 \%(\mathrm{n}=117)$ of participants reported being comfortable getting an HIV self-test kit by mail or for home use from a clinic.

Conclusion Our findings show the potential utility for home HIV self-testing to enhance HIV serostatus awareness in Peru. 